\title{
High Resolution and Wide Dynamic Range Pressure Sensor Based on Two-Dimensional Photonic Crystal
}

\author{
Saeed OLYAEE and Ali Asghar DEHGHANI
}

\author{
Nano-photonics and Optoelectronics Research Laboratory (NORLab), Faculty of Electrical and Computer Engineering, \\ Shahid Rajaee Teacher Training University (SRTTU), Tehran, Iran \\ *Corresponding Author: Saeed OLYAEE_ E-mail: s_olyaee@srttu.edu
}

\begin{abstract}
The authors present a new design of high resolution and wide dynamic range photonic crystal pressure sensor. This sensor is based on two-dimensional photonic crystal with square array of silicon rods surrounded by air. The sensor consists of a photonic crystal waveguide which is coupled to a photonic crystal nanocavity. The waveguide is configured by removing one row of $\mathrm{Si}$ rods and nanocavity is formed by modifying the radius of one Si rod. The sensor is designed for $1300 \mathrm{~nm}-1400 \mathrm{~nm}$ wavelengths. Simulation results show that resonant wavelength of nanocavity is linearly shifted to larger wavelengths by increasing the pressure. The designed sensor has a linear behavior between $0.1 \mathrm{GPa}$ to $10 \mathrm{GPa}$ of applied pressure and $8 \mathrm{~nm} / \mathrm{GPa}$ of pressure sensitivity.
\end{abstract}

Keywords: Photonic crystal, waveguide, nanocavity, pressure sensor

\section{Introduction}

Since the last decade, photonic crystals (PhCs) have been attractive optional structures for controlling and manipulating the light. The PhCs are the periodic microstructures with a period in order of optical wavelength that the refractive index of material changes in one, two, or three directions. Their inherent properties, such as photonic band gap (PBG), reflectance-transmittance, high design flexibility, and possibility to be made in nanometer range, make it possible to utilize these structures in many applications such as waveguides, nano-resonators, photonic crystal fibers [1-5]. The research fields of PBG structures are explosively growing, with numerous novel applications being introduced every day [6].

Their application as sensor is a new research field that seems to be applicable in nano-electro-mechanical systems (NEMS). Xu et al. have proposed and analyzed a micro-displacement sensor with a large dynamic range based on a two-dimensional (2D) PhC co-directional coupler, by which the sensing range can be as large as tens of lattice constant or even more [7]. Shi et al. have reported an optical humidity sensor based on a nano-porous polymeric $\mathrm{PhC}$ that have excellent reversibility and reproducibility [8]. Other works such as refractive index sensor [9], chemical sensor, and oil sensor [10] have been also reported.

The $\mathrm{PhC}$ pressure sensor can be constructed by coupling a waveguide and a nanocavity. A waveguide is made by introducing line defect in 2D $\mathrm{PhC}$ structure, and a nanocavity is made by introducing point defect in $\mathrm{PhC}$ structure.

The resonant wavelength of nanocavity is a function of shape, dimension, and surface state of defects. This point is a fundamental work of pressure sensor. When pressure is applied to the nanocavity, its characteristics change, and therefore

Received: 15 August 2011 / Revised version: 7 October 2011

CThe Author(s) 2011. This article is published with open access at Springerlink.com 
the linear relation between pressure and resonant wavelength shift can be observed $[11,12]$. In this paper, we propose a novel, linear, and high resolution pressure sensor.

\section{Principle of the pressure sensor}

The effect of hydrostatic pressure on the electronic and optical properties of material such as energy gap and refractive index can be considered for sensing applications. When a crystal is compressed by the pressure, the band gap is increased. The refractive index of $\mathrm{Si}$ is modified when optical coefficients such as photoelastic, piezoelectric, and permittivity change in different strains. In PhC structure, PBG is dependent on refractive index, lattice constant, and radius-tolattice constant ratio $\left(\frac{r}{a}\right)$. By applying the pressure to the $\mathrm{PhC}$, the refractive index of material, the geometrical shape of $\mathrm{PhC}$, and the PBG of structure change. In the $\mathrm{PhC}$ waveguide coupled to the nanocavity output, the spectrum of waveguide changes with different strains. On the other hand, resonant wavelength of nanocavity is dependent on the geometrical shape of defect that makes the cavity. By applying certain pressure to the structure, the resonant wavelength and resonant wavelength shift of nanocavity can be measured as a function of pressure. This point is a fundamental idea for $\mathrm{PhC}$ pressure sensor. In this section, we calculate the refractive index modulation resulted from the applied pressure. To determine the refractive index of the stressed structure, we utilize optical tensor coefficients for silicon characterized by the stress-optical tensor equation $[6,13]$ :

$$
\left[\begin{array}{l}
n_{x x} \\
n_{y y} \\
n_{z z} \\
n_{y z} \\
n_{x z} \\
n_{x y}
\end{array}\right]=\left[\begin{array}{l}
n_{0} \\
n_{0} \\
n_{0} \\
0 \\
0 \\
0
\end{array}\right]-\left[\begin{array}{cccccc}
c_{1} & c_{2} & c_{2} & 0 & 0 & 0 \\
c_{2} & c_{1} & c_{2} & 0 & 0 & 0 \\
c_{2} & c_{2} & c_{1} & 0 & 0 & 0 \\
0 & 0 & 0 & 0 & 0 & 0 \\
0 & 0 & 0 & 0 & 0 & 0 \\
0 & 0 & 0 & 0 & 0 & 0
\end{array}\right]\left[\begin{array}{l}
\sigma_{x x} \\
\sigma_{y y} \\
\sigma_{z z} \\
\sigma_{y z} \\
\sigma_{x z} \\
\sigma_{x y}
\end{array}\right]
$$

where $n_{i j}$ denotes the refractive index along $i j$ direction, $n_{0}$ is the refractive index in zero-pressure, and $\sigma_{i j}$ is the pressure along $i j$ direction. We assume that pressure is applied in one direction, therefore:

$$
\begin{gathered}
\sigma_{x y}=\sigma_{x z}=\sigma_{y z}=0 \\
\sigma_{x x}=\sigma_{y y}=\sigma_{z z}=\sigma .
\end{gathered}
$$

Thus pressure-modified refractive index value reduces to:

$$
n=n_{0}-\left(c_{1}+2 c_{2}\right) \sigma
$$

where $c_{1}$ and $c_{2}$ are defined as

$$
\begin{gathered}
c_{1}=\frac{n_{0}^{3}\left(P_{11}-2 V P_{12}\right)}{2 E} \\
c_{2}=\frac{n_{0}^{3}\left[\left(P_{12}-V\left(P_{11}+P_{12}\right)\right]\right.}{2 E}
\end{gathered}
$$

where $E$ is Young's modulus, $V$ is Poisson's ratio, and $P_{i j}$ denotes strain-optic constant. For silicon at $\lambda=1.3 \mu \mathrm{m}$ we have

$$
P_{11}=-0.101, P_{12}=-0.0094 \text {. }
$$

\section{Design of $\mathrm{PhC}$ sensor structure}

Waveguide is an important element in optical circuit to conduct light between components. The researches mainly focus on the waveguides with only a few resonant electromagnetic modes, because of drastically reducing the size of photonic components. In 2D PhC waveguide, a linear defect is introduced into the crystal, creating a localized band that falls within and is guided by the photonic band gap [14]. The waveguide can be made by removing one row of air holes or by eliminating one row of dielectric rods. The wave propagates in $\mathrm{Si}$ for the first case and propagates in air for the second case, and consequently, the loss can be ignored. This waveguide is constructed in silicon-on-insulator (SOI) wafer. In SOI wafer having $\mathrm{Si}$ rods, $\mathrm{Si}$ and $\mathrm{SiO}_{2}$ are the substrate $(n=3.5)$ and the insulator $(n=1.45)$, respectively, and Si rods are configured in $\mathrm{Si}$ top layer [15]. The $\mathrm{PhC}$ nanocavity resonator with a high quality factor $(Q)$ can be used in many applications. The $Q$ value of nanocavity can be improved by changing the number of crystal periods between the nanocavity and the waveguide.

In this research, the designed photonic crystal 
structure consists of a square array of Si rods with $n=3.5$ surrounded by air with $n=1$. The lattice constant and radius of $\mathrm{Si}$ rods are respectively considered as $\alpha=450 \mathrm{~nm}$ and $r=0.2 a$. By performing plane wave expansion (PWE) approach, the $\mathrm{PhC}$ indicates normalized frequency of photonic band gap for TE mode between $0.2795 \mathrm{eV}$ and $0.4154 \mathrm{eV}$ that is equal to wavelengths between $1083 \mathrm{~nm}$ and $1610 \mathrm{~nm}$.

Figure 1 shows the layout of the designed photonic crystal sensor. In this paper, we optimize the $\mathrm{PhC}$ waveguide and nanocavity to be applied in near infrared from $1300 \mathrm{~nm}$ to $1400 \mathrm{~nm}$. The $\mathrm{PhC}$ waveguide is formed by removing one row of $\mathrm{Si}$ rods in the PhC, and nanocavity is formed by modifying the radius of one Si rod to $R_{c}=0.077 a$. In this study, there are two crystal rods between nanocavity and waveguide that results in a good quality factor for nanocavity. The length and width of this sample are respectively designed as $l=9.2 \mu \mathrm{m}$ and $w=4.7 \mu \mathrm{m}$.

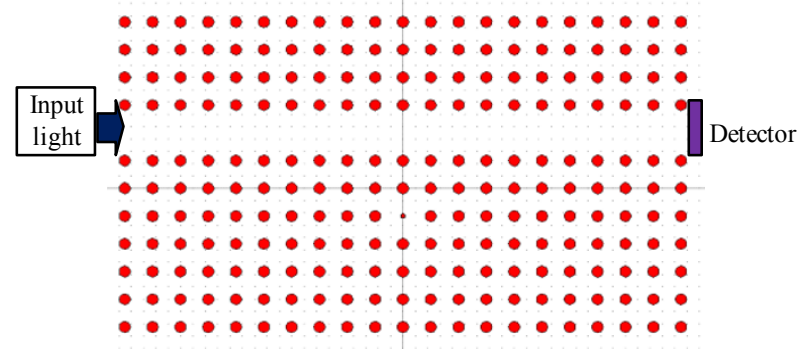

Fig. 1 Layout of sensor structure consisting of a square array of Si rod in air with $a=450 \mathrm{~nm}$ and $r=0.2 a$.

\section{Simulation results}

The two-dimensional finite-difference time-domain (2D-FDTD) method is applied to simulate propagation of electromagnetism wave in waveguide. The structure is excited by Gussian pulse and by applying appropriate boundary condition (perfectly matched layer, PML). As shown in Fig. 2, in absence of pressure, the nanocavity shows resonant wavelength at $\lambda=1316 \mathrm{~nm}$. The quality factor for nanocavity is $Q=1470$. The structure is excited by continuous wave $(\mathrm{CW})$ to simulate the field pattern in the waveguide. Figure 3 illustrates the simulation result of light propagation through the pressure sensor. The interaction between the wave propagated in the waveguide and field localized in the nanocavity results in the change in the transmission spectrum from the cavity to the end of waveguide, and the field intensity passing through the nanocavity reduces.

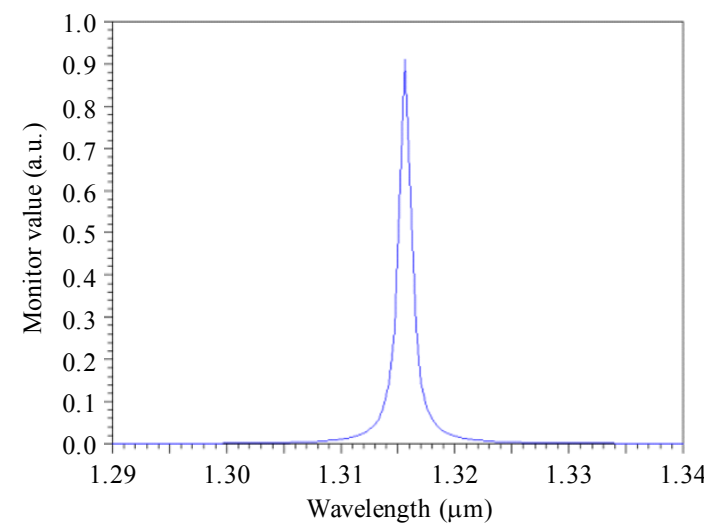

Fig. 2 Transmission spectrum of the cavity without applied pressure (the resonant wavelength is $\lambda=1316 \mathrm{~nm}$ ).

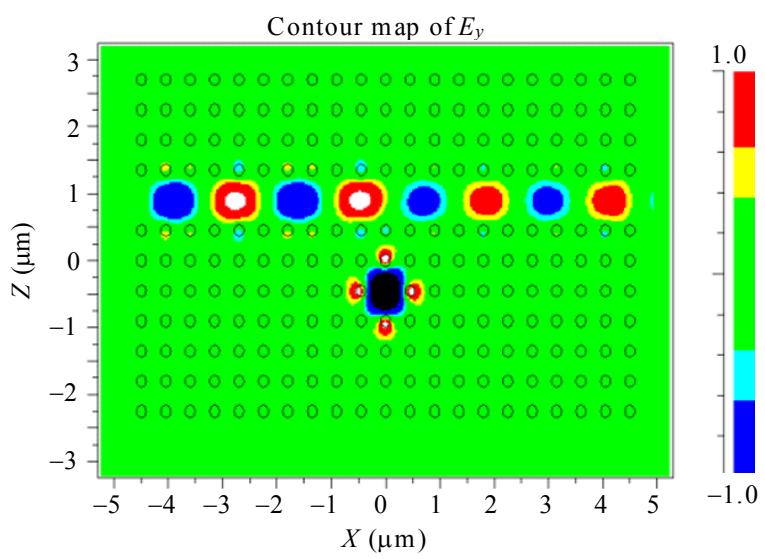

Fig. 3 Simulation result of light propagation in the structure.

To analyze pressure effect on the structure, we perform comprehensive simulation study. The simulations have been performed in different pressures from 0 to $10 \mathrm{GPa}$ by $1-\mathrm{GPa}$ increment at each step, and corresponding resonant wavelengths obtained with different curves are shown in Fig. 4. It has been observed that with increment in the refractive index, the resonant wavelength shifts to larger wavelength region. For example, in 1-GPa pressure, the refractive index of $\mathrm{Si}$ changes from 
3.50 at zero pressure to 3.5398 , and consequently the resonant wavelength shifts from $1316 \mathrm{~nm}$ to $1324 \mathrm{~nm}$. In 2-GPa pressure, the refractive index is calculated as 3.5797, and nanocavity resonant wavelength is obtained to be $1332 \mathrm{~nm}$.

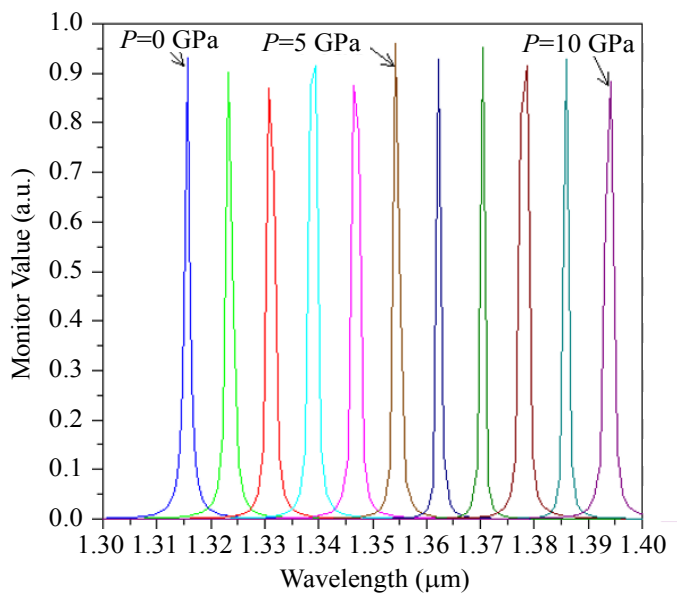

Fig. 4 Transmission spectra for different pressures from 0 to $10 \mathrm{GPa}$.

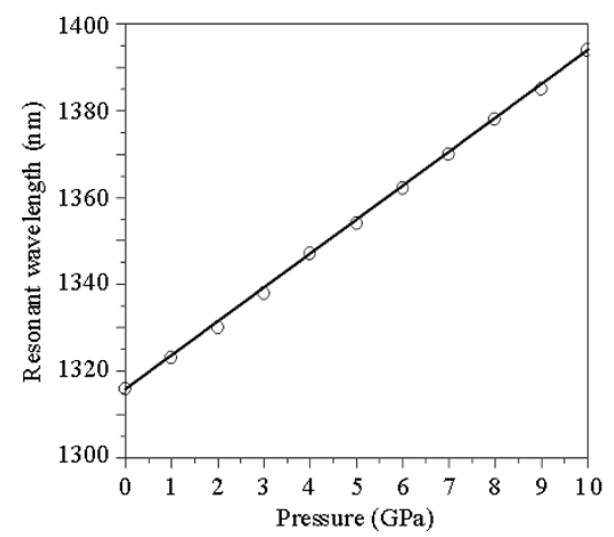

Fig. 5 Linear relationship between resonant wavelength and pressure in the range of 0 to $10 \mathrm{GPa}$.

By analyzing the results of Fig. 4, it can be shown that there is a linear relationship between applied pressure and resonant wavelength. Therefore, we can estimate the sensitivity of sensor equal to $8 \mathrm{~nm} / \mathrm{GPa}$, which shows better result compared to well-known researches, e. g. in [11]. Since the resonant peak has a very narrow linewidth, the resolution can be quite high. Figure 5 indicates an excellent linear relationship between pressure and resonant wavelength shift. This curve also indicates a wide dynamic range for this sensor from 0 to 10-GPa pressure. By numerical analysis, the minimum detectable pressure with this structure is $3 \mathrm{mN}$. With $0.1-\mathrm{GPa}$ incremental pressure, nanocavity shows $0.9-\mathrm{nm}$ increment in resonant wavelength, as shown in Fig. 6.

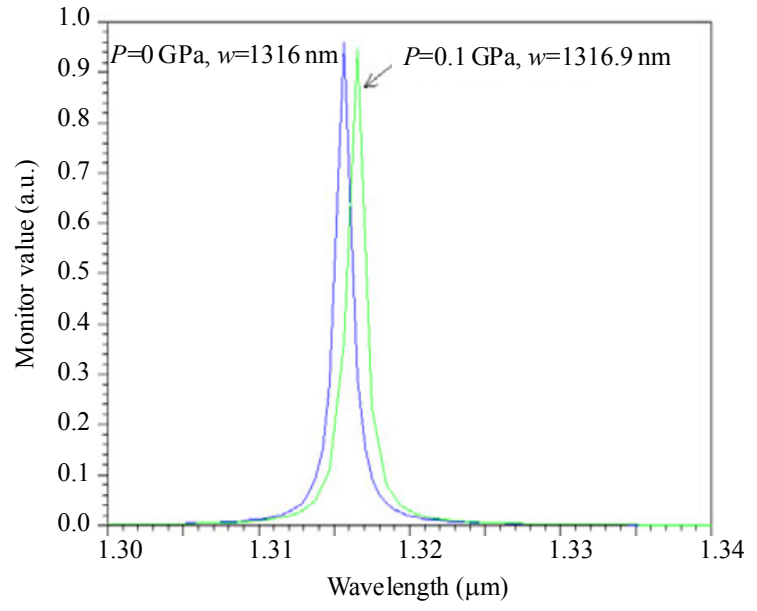

Fig. 6 Resonant wavelength for nanocavity by $0.1-\mathrm{GPa}$ increment in pressure.

\section{Conclusions}

In this paper, a novel photonic crystal pressure sensor has been designed and presented. This sensor is constructed with two-dimensional photonic crystal waveguide which is coupled to the nanocavity. By changing optical properties of $\mathrm{Si}$, the refractive index changes, and therefore, resonant wavelength of nanocavity shifts. With emphasis on this point, the sensor can be calibrated to measure applied pressure. The designed sensor has a good resolution in $\mathrm{mN}$ range, quality factor equal to 1470 , and wide linearity range between 0 to $10 \mathrm{GPa}$. By applying similar scheme and improving quality factor of nanocavity, the resolution of senor can be improved. This sample can be utilized in nanotechnology and NEMS industry.

\section{Acknowledgment}

The authors would like to thank Iran Nanotechnology Initiative Council (INIC) for financial support.

Open Access This article is distributed under the terms of the Creative Commons Attribution License which 
permits any use, distribution, and reproduction in any medium, provided the original author(s) and source are credited.

\section{References}

[1] J. D. Joannopoulos, S. G. Johnson, J. N. Winn, and R. D. Meade, Photonic crystals molding the flow of light. New Jersey, USA: Princeton University Press (ISBN: 978-0-691-12456-8), 2007.

[2] S. Olyaee and F. Taghipour, "Design of new square-lattice photonic crystal fibers for optical communication applications," Int. J. Physical Sci., vol. 6, no. 18, pp. 4405-4411, 2011.

[3] M. Fatih and Y. Fan, "High-contrast all-optical bistable switching in photonic crystal microcavities," Appl. Phys. Lett., vol. 83, no. 14, pp. 6, 2003.

[4] S. Olyaee and F. Taghipour, "Ultra-flattened dispersion photonic crystal fiber with low confinement loss," in the 11th International Conference on Telecommunications, ConTEL, Graz University of Technology, Austria, June 15-17, pp. 531-534, 2011.

[5] S. Olyaee and F. Taghipour, "A new design of photonic crystal fiber with ultra-flattened dispersion to simultaneously minimize the dispersion and confinement loss," Journal of Physics: Conference Series, The 3rd International Photonics and OptoElectronics Meeting (POEM2010), Wuhan, China, vol. 276, no. 1, pp. 012080, 2011.

[6] K. Radulović, Z. Jakšić, and Z. Djurić, "Design and simulation of stressed 2D photonic crystals by finite element method," in Proc. II International Symposium Light Metals and Composite Materials, Belgrade, May 19-20, pp. 99-101, 2004.
[7] Z. Xu, L. Cao, P. Su, Q. He, G. Jin, and G. Gu, "Micro-displacement sensor with large dynamic range based on photonic crystal co-directional coupler," IEEE Journal of Quantum Electronic, vol. 43, no. 2, pp.182-186, 2007.

[8] J. Shi, V. S. Hsiao, T. R. Walker, and T. J. Huang, "Humidity sensing based on nanoporous polymeric photonic crystals," Sensors and Actuators B, vol. 129, no. 1, pp. 391-396, 2008.

[9] J. Sun and C. C. Chan, "Photonic bandgap fiber for refractive index measurement," Sensors and Actuators $B$, vol. 128, no. 1, pp. 46-50, 2007.

[10] R. V. Nair and R. Vijaya, "Photonic crystal sensors: an overview," Progress in Quantum Electronics, vol. 34, no. 3, pp. 89-134, 2010.

[11] T. Stomeo, M. Grande, A. Qualtieri, A. Passaseo, A. Salhi, M. De Vittorio, and D. Biallo, "Fabrication of force sensors based on two-dimensional photonic crystal technology," Microelectronic Engineering, vol. 84, no. 5-8, pp. 1450-1453, 2007.

[12] T. T. Mai, F. Hsiao, C. Lee, W. Xiang, C. Chen, and W. K. Choi, "Optimization and comparison of photonic crystal resonators for silicon microcantilever sensor," Sensors and Actuators A, vol. 165, no. 1, pp.16-25, 2011.

[13] M. Huang, "Stress effects on the performance of optical waveguides," Internat. J. Solids and Struct., vol. 40, no. 7, pp. 1615-1632, 2003.

[14] S. G. Johnson, P. R. Villeneuve, S. Fan, and J. D. Joannopoulos, "Linear waveguides in photonic-crystal slabs," Physical Review B, vol. 62, no. 12, pp. 8212-8222, 2000.

[15] M. Loncar, T. Doll, J. Vuckovic, and A. Scherer, "Design and fabrication of silicon photonic crystal optical waveguides," J. Lightwave Technology, vol. 18, no. 10, pp. 1402-1411, 2000. 